\title{
Gradhiva
}

GRADHIV

Revue d'anthropologie et d'histoire des arts

$1 \mid 2005$

Haïti et l'anthropologie

\section{Cheminement anthropologique en Haïti}

Carlo Avierl Célius

\section{OpenEdition}

Journals

Édition électronique

URL : http://journals.openedition.org/gradhiva/263

DOI : 10.4000/gradhiva.263

ISSN : 1760-849X

\section{Éditeur}

Musée du quai Branly Jacques Chirac

\section{Édition imprimée}

Date de publication : 1 mai 2005

Pagination : 47-55

ISBN : 2-915133-08-5

ISSN : 0764-8928

\section{Référence électronique}

Carlo Avierl Célius, « Cheminement anthropologique en Haïti », Gradhiva [En ligne], 1 | 2005, mis en ligne le 10 décembre 2008, consulté le 21 décembre 2020. URL : http://journals.openedition.org/ gradhiva/263; DOI : https://doi.org/10.4000/gradhiva.263

Ce document a été généré automatiquement le 21 décembre 2020.

(c) musée du quai Branly 


\title{
Cheminement anthropologique en Haïti
}

\author{
Carlo Avierl Célius
}

1 L'anthropologie "apparaît comme étant consubstantielle à l'histoire d'Haïti ", parce que «les événements qui ont engendré l'Indépendance du pays semblaient être le produit d'une lutte de races $»^{1}$. Cette racialisation des faits se comprend dans un cadre paradigmatique élargi, à saisir tant dans la synchronie que dans la diachronie. Le développement, dans la colonie française de Saint-Domingue au $\mathrm{XVIII}^{\mathrm{e}}$ siècle, de la grande plantation dévolue à la monoculture sucrière s'accompagne de la bipolarisation de la société coloniale selon le schéma maître blanc / esclave noir. La segmentation raciale est alors juxtaposée à la stratification socio-économique, déjà sanctionnée par une coupure juridique (le Code noir, 1685) ${ }^{2}$. Ainsi « la "race" finit par
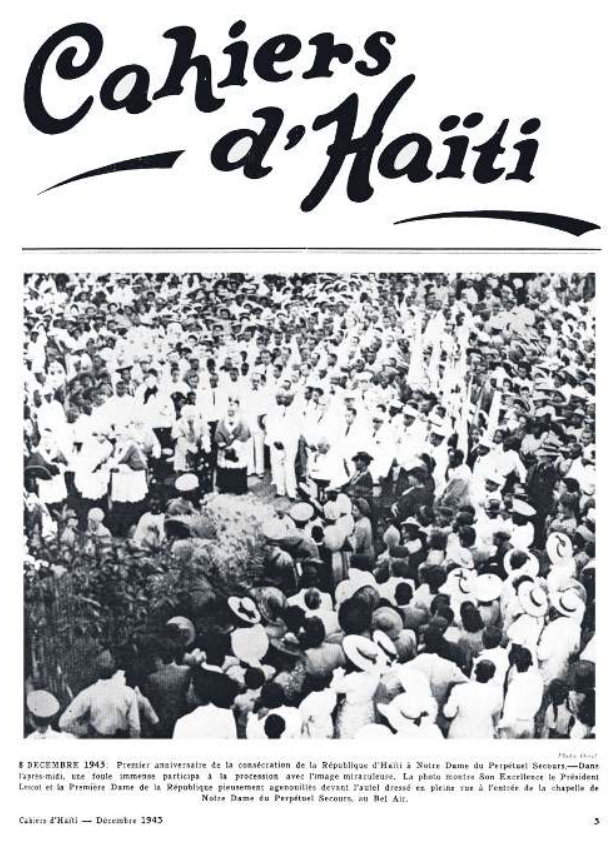
devenir consubstantielle à l'ordre esclavagiste» qui mérite en conséquence le qualificatif de socio-racial ${ }^{3}$. La question raciale situe donc le rapport à l'anthropologie en amont des événements ayant abouti à 1804. Et comme le discours anthropologique commence à s'élaborer à partir des premiers contacts des Européens avec le Nouveau Monde, il s'ensuit que la Caraïbe, où se sont effectués ces premiers contacts, en constitue un des lieux fondateurs ${ }^{4}$.

2 Advenus simultanément, l'espace caraïbe reconfiguré et le discours anthropologique nouent des liens originels et interféconds. Pourtant devenue discipline autonome, l'anthropologie entretiendra des rapports difficiles avec la région, apparue comme « undisciplined », dont les formes d'expériences défient ses postulats de base. La pureté 
et l'anhistoricité y sont introuvables du fait de l'hétérogénéité constitutive des sociétés caraïbes prises dans une dynamique continue. D'où un hiatus, que tendent à combler de nouvelles théories, entre les approches internes qui ont toujours cherché à penser ces réalités et les courants jusque-là dominants de l'anthropologie. Il est donc impératif de s'efforcer de tenir ensemble, dans un va-et-vient incessant, les discours autochtones et allogènes sur la région, confrontés aux grands courants de pensée, pour saisir les jeux et enjeux des modes d'appropriation, de réélaboration, d'opposition, dans la production anthropologique de l'objet Caraïbe.

De telles considérations remettent en question une périodisation couramment admise fixant le début de la réflexion anthropologique en Haïti (ou à partir d'Haïti), non pas en 1804, mais en 1885 avec la parution de De l'égalité des races humaines d'Anténor Firmin, suivie d'un tournant ethnologique amorcé en 1928 par Jean Price-Mars avec Ainsi parla l'oncle. Ces œuvres sont des moments de cristallisation, dont la portée se mesure par rapport à toute une sédimentation intellectuelle qu'elles remuent, bouleversent, reconfigurent et enrichissent, mais qui les porte. En établissant une stratigraphie descendant jusqu'au Journal de bord de Christophe Colomb, Catts Pressoir, Ernst et Hénock Trouillot en font la démonstration pour l'Historiographie d'Haïti'. Mais il faut surtout être attentif aux maillages intertextuels de la production intellectuelle d'Haïti pour prendre la mesure du labourage sans cesse à l'œuvre. Le temps colonial reste le socle à partir duquel on se positionne. Avec une phase déterminante, la deuxième moitié du XVIII ${ }^{\mathrm{e}}$ siècle, marquée surtout par la création d'une société savante, le Cercle des Philadelphes, qui entreprend d'organiser la production des connaissances dans et sur la colonie ${ }^{7}$. Moreau de Saint-Méry passe pour le plus connu de cette équipe, avec une œuvre d'où se détache sa célèbre Description... de Saint-Domingue ${ }^{8}$. CEuvre encyclopédique où puisent toutes les disciplines, depuis le $\mathrm{XIX}^{\mathrm{e}}$ siècle, elle demeure une des pièces maîtresses de l'anthropologie de la période coloniale, et son auteur la figure dominante de «l'ethnographie de la communauté dominguoise transformée en communauté haïtienne $»^{9}$. 


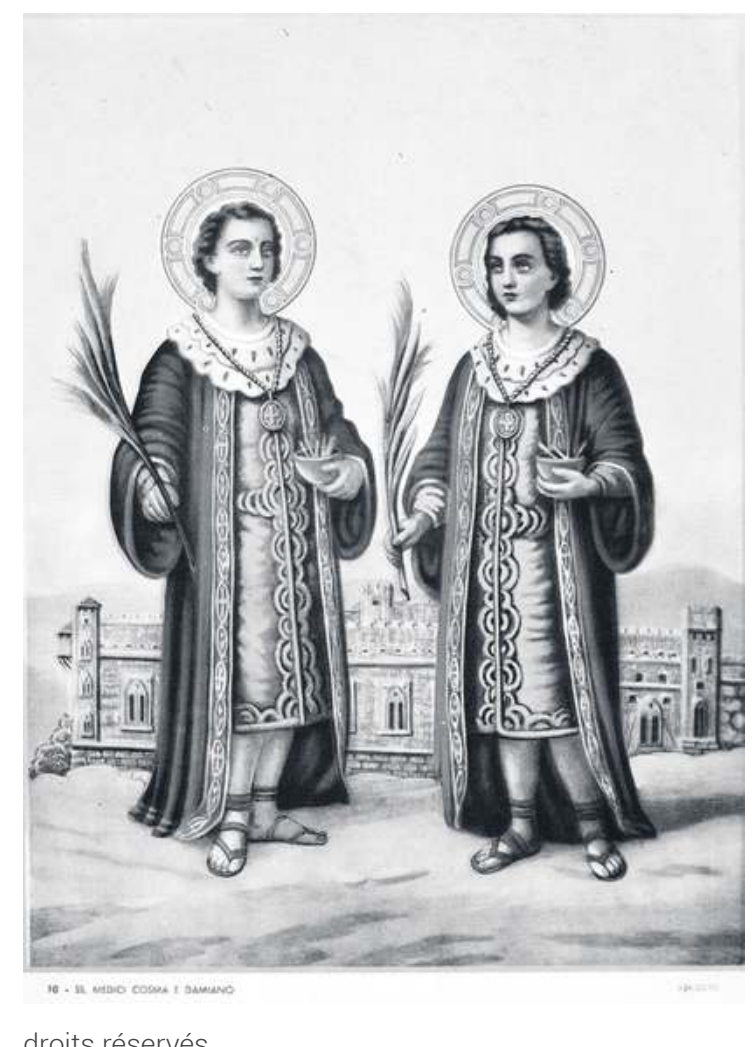

4 Cependant, les conditions dans lesquelles s'est effectué le passage d'une société coloniale à une société indépendante, la naissance d'un nouveau corps politique, ont rendu possible et nécessaire la formulation d'un projet anthropologique réfutant bien des aspects du discours qui justifiait l'ordre ancien. Ce projet, Thomas Madiou le résume, en 1847, dans ce passage de l'introduction à son Histoire d'Haïti ${ }^{10}$ :

«Cette race, sortant de l'Afrique, plus vigoureuse que celle des Indiens, soumise aussi à l'esclavage, brisera ses chaînes et formera une nation nouvelle. Elle reprendra le nom des Aborigènes, et, tout en fondant une patrie libre pour la Race Africaine, elle fera expier aux peuples de l'Europe, et sa servitude, et la destruction des Indiens. Elle sera une nouvelle preuve que certaines parties de la terre ne sont pas plus que d'autres le domaine de quelques espèces de la race humaine. À la race humaine appartient le globe entier : un peuple succède à un peuple, une race à une race. Les hommes forment des courants qui se croisent dans toutes les directions. Dans les contrées qu'occupent les nations d'aujourd'hui, on rencontre peu de populations qui soient aborigènes; car en remontant dans l'histoire, nous ne voyons qu'émigration et transplantations. L'esprit humain ne progresse que par ce frottement, cette fusion des races. »

5 Ces réflexions, qui mériteraient un très long commentaire, parcourent bien des écrits des quarante premières années du XIX ${ }^{\mathrm{e}}$ siècle. La notion de race, employée confusément, est centrale. Elle sert à caractériser l'indépendance qui, bien que localisée et expliquée par des causes particulières, participe d'une dynamique générale d'occupation du globe par «la race humaine ». En fin de compte, une chose est essentielle pour Madiou : l'homme est acteur de l'histoire, laquelle est conçue comme un processus continu d'humanisation. 
Universalisation (des faits, des pratiques, des événements historiques) vaut humanisation - un double procès dans lequel se placent Haïti et son peuple. Cette perspective prend racine dans un acte fondateur : le renversement du système esclavagiste. La conquête de la liberté invalide tout le dispositif discursif qui avait été construit pour légitimer sa négation et le corollaire de celle-ci, la déshumanisation. Elle rend énonçable un contrediscours visant à « dé-naturaliser » l'esclavage et à défendre l'humanité de tout homme. Dans un monde alors et pour longtemps encore esclavagiste, colonialiste et raciste, c'est bien d'un contre-discours qu'il s'agit.

D'où, dans une pensée haïtienne en éveil, des discussions inlassables sur l'égalité des races, sur l'aptitude de la race noire à «La Civilisation », sur la preuve de cette aptitude par l'existence d'Haïti, sur l'état d'avancement de ce processus dans le pays, sur la réversibilité d'un tel processus, illustrée par l'histoire de l'Afrique, sur le rôle d'Haïti dans la régénération de la race... C'est-à-dire une pensée de combat, manifeste jusque dans la conception et la pratique des beaux-arts ainsi que dans le discours esthétique, à travers la thématique de la beauté dans les races (Célius, pp. 71-94). Autant de questions retravaillées par Anténor Firmin dans De l'égalité des races humaines, à partir d'une "Anthropologie positive» fondée sur une critique systématique de la raison anthropologique dans ses élaborations les plus avancées à la fin du XIX ${ }^{e}$ siècle.

Haïti 1915 : prisonniers

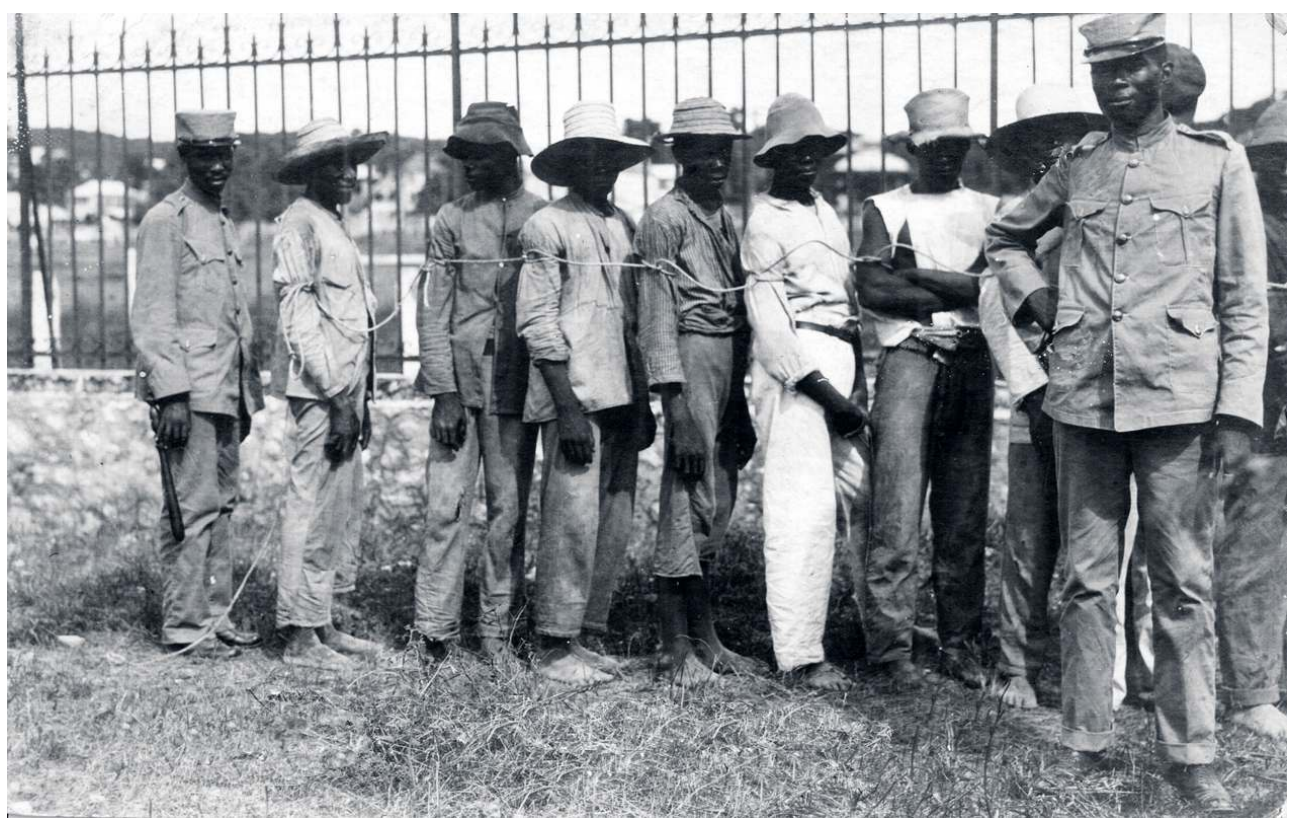

(C) Archives CIDIHCA

À propos de cette œuvre, préparée par un Firmin alors membre de la Société d'anthropologie de Paris et autour de laquelle s'est tissé un voile de silence ${ }^{11}$, Carolyn Fluehr-Lobban ${ }^{12}$ nous montre qu'elle a sa place parmi les principaux textes fondateurs de l'anthropologie (pp. 95-108) - ceux de Lewis H. Morgan (Ancient Society, 1877), de Edward B. Tylor (Anthropology, 1881), de Paul Topinard (Éléments d'anthropologie générale, 1885), de Franz Boas (The Mind of Primitive Man, 1911) ${ }^{13}$. Firmin, s'appuyant sur le positivisme d'Auguste Comte, revisite l'histoire de la tradition philosophique et scientifique pour déconstruire toutes les thèses sur lesquelles repose l'idéologie de l'inégalité des races 
humaines. Ce qui l'amène à traiter des conditions de production de la connaissance scientifique, à s'interroger sur les logiques de pouvoir à l'œuvre dans l'élaboration des savoirs et à anticiper bien des propositions formulées par la suite par des chercheurs comme Durkheim, Boas, entre autres. Fluehr-Lobban mène une longue comparaison entre Firmin et Price-Mars pour montrer la continuité entre les deux penseurs et l'impact du premier sur l'anthropologie afro-américaniste par le biais du second.

Kevin Yelvington et Gérarde Magloire (pp. 127-152), dans un même souci de tisser cette ligne de continuité, rejoignent C. Fluehr-Lobban. Si la continuité est en effet indéniable, elle ne résorbe pas la spécificité de l'apport respectif de Firmin et de Price-Mars. Elle ne doit surtout pas occulter d'autres contributions déterminantes dans l'élaboration de l'œuvre de Price-Mars. Roger Gaillard, dans sa présentation à la dernière édition de Ainsi parla l'oncle rappelle que le retentissement immédiat de l'ouvrage tient au fait que le terrain avait été largement balisé, au moins dans les domaines littéraire et artistique ${ }^{14}$. Ainsi Ghislain Gouraige avait déjà relevé la dette contractée par Price-Mars à l'endroit des réflexions et des œuvres littéraires de Frédéric Marcelin, fin XIX ${ }^{\mathrm{e}}$-début $\mathrm{XX}^{\mathrm{e}}$ siècle ${ }^{15}$. PriceMars a payé son dû à Antoine Innocent, qui, selon le poète Carl Brouard, est celui qui a introduit le vodou dans la littérature haïtienne avec son roman Mimola $(1906)^{16}$. Parmi toutes les autres références identifiables, il y a lieu de s'attarder un peu sur l'une d'entre elles, souvent évoquée trop rapidement pour mieux mettre en relief ce qui serait la contribution décisive de Price-Mars.

En 1912-1913, le Dr J. C. Dorsainvil publie une série d'articles et une étude de 1924, réunies par la suite en un volume, sous le titre Vodou et névrose ${ }^{17}$. Dans l'introduction de son ouvrage, l'auteur soutient et réaffirme que la possession «n'est que le domaine pathologique du sentiment religieux et de la croyance ${ }^{18}$. Price-Mars objecte que la possession ne relève pas de la pathologie. Mais on oublie que Dorsainvil soutient par ailleurs que le vodou est une religion. Pourtant il insiste : «La possession est intimement liée à l'histoire des religions. Elle est, comme nous l'avons dit, une manifestation morbide du sentiment religieux ${ }^{19}$. Ce n'est pas la seule proposition de l'auteur. Homme de terrain ${ }^{20}$, il mène des enquêtes dont il entend rendre compte des résultats scientifiquement. Il soutient que le vodou se perpétue "par la tradition familiale » et qu'« il existe dans le pays une mentalité vôdouique à laquelle peu d'entre nous échappent $»^{21}$. D'où la nécessité de se pencher sur cet aspect de notre culture: "Un peuple ne saurait en effet se désintéresser de ce problème. À le méditer attentivement, il y découvre souvent les motifs de ses qualités comme les raisons de ses défauts. C'est de la psychologie rétrospective, vrai coup de sonde jeté dans l'abîme des temps passés. » Il martèle : « Nous le soutenons avec conviction. On ne peut pénétrer intimement la mentalité du peuple haïtien, sans une étude approfondie de nos antiquités africaines. Cette étude doit se poursuivre à la fois dans les domaines physiologique, historique, psychologique et moral. $»^{22}$ Car « elle n'est [...] pas viable, la race ou la nation qui, parvenue à une certaine civilisation, méprise son passé comme indigne d'elle, parce qu'alors, elle aura rompu sous ses pas la chaîne des traditions qui la lie avec ses ancêtres ». Et Dorsainvil s'étonne « de constater à quel degré, nos historiens et nos sociologues, dans l'étude des dominantes de notre évolution historique, ont complètement négligé l'Afrique »"3. Pourtant Haïti « n'est qu'un prolongement de l'Afrique dans l'espace $»^{24}$. C'est dans cette perspective que l'auteur aborde la langue créole. À partir d'« une explication philologique du Vodou », il en vient à la conclusion que «quoique constitué de mots français déformés, il [le créole] est coulé dans le moule racial. Il fait un usage abusif des affixes nasalisés qui caractérisent 
les idiomes dahoméens et congolais » ${ }^{25}$. Précision : " Nous avons conservé dans le créole, le cadre, la structure de la phrase dahoméenne ou congolaise, ce que nous avons appelé plus haut le moule racial ${ }^{26}$. À noter qu'il insiste sur l'apport du «fongbe, un des six dialectes ewe ». Dorsainvil invitait à étudier de manière approfondie cette question. Suzanne Comhaire-Sylvain, qui deviendra une figure éminente de l'école haïtienne d'ethnologie ${ }^{27}$, entendra l'appel et reformulera sa proposition dans une phrase restée célèbre: "Nous sommes en présence d'un français coulé dans le moule de la syntaxe africaine ou, comme on classe en général les langues d'après leur parenté syntaxique, d'une langue ewe à vocabulaire français $\aleph^{28}$. En fait, plus qu'une phrase, ce sont les bases de tout un courant de la créolistique, le courant dit afrogénétique ${ }^{29}$, qui sont alors posées. On notera aussi que Dorsainvil inaugure tout un pan de la recherche qui articule l'ethnologie à la psychologie, à la psychanalyse, à la psychiatrie, et qui est notamment illustrée par les travaux de Louis Mars, Emerson Douyon, Jeanne Philippe, Willy Apollon ${ }^{30}$. Louis Mars a écrit, de l'aveu de Georges Devereux, la première synthèse de psychiatrie comparée ${ }^{31}$, avant d'être l'inventeur - aujourd'hui oublié - de l'ethnopsychiatrie.

11 Une réévaluation globale de l'apport de Dorsainvil devrait être faite et, dans cette optique, entreprise une lecture comparative approfondie de Vodou et névrose et de Ainsi parla l'oncle. On perçoit tout de même déjà que Dorsainvil énonce l'essentiel de ce que formulera Price-Mars : le statut de religion du vodou, sa prégnance dans la société et la nécessité de l'étudier, la critique du refus des élites de reconnaître et de valoriser les héritages africains... Price-Mars admettra en 1954 que Dorsainvil «introduisit les éléments basiques des investigations ultérieures $»^{32}$. Avec une différence essentielle tenant aux approches liées à leurs disciplines respectives de référence. Dorsainvil dit pratiquer la "médico-sociologie ", il se préoccupe plus particulièrement des problèmes d'ordre psychologique et psychopathologique, qui intéressent également Price-Mars, mais il les intègre à une démarche et à une discipline que Dorsainvil ne nomme même pas, l'ethnographie et l'ethnologie.

12 La différence entre ces deux notions est établie par Firmin dès 1885. La même année D. Trouillot publie à Port-au-prince Esquisse ethnographique. Le Vaudoun: aperçu historique et évolutions ${ }^{33}$. Il cherche à accréditer l'idée, alors répandue, d'un culte insignifiant en voie de disparition accélérée, mais parvient à poser les fondements d'une ethnographie du vodou. Il tente de fixer spatialement et temporellement son apparition, tout en faisant ressortir le caractère «universel» de certaines formes de croyances et de pratiques rituelles qu'il recèle (usage des pierres de foudre, sacrifice animal, culte "disparu» du serpent...). Il propose une description des cérémonies d'initiation, dresse un tableau des lwa du panthéon et du vocabulaire vodou. Il établit des distinctions essentielles entre le vodou - qu'il est peut-être l'un des premiers à qualifier de "syncrétique »- la sorcellerie et la médecine traditionnelle. Il relève des tabous alimentaires liés aux pratiques cultuelles. Cependant, à la question essentielle : le vodou est-il pour lui une religion ? La réponse est ambivalente. Il y voit de la superstition, mais soutient qu'on a affaire à une religion qui n'existerait plus en tant que telle en Haïti. 


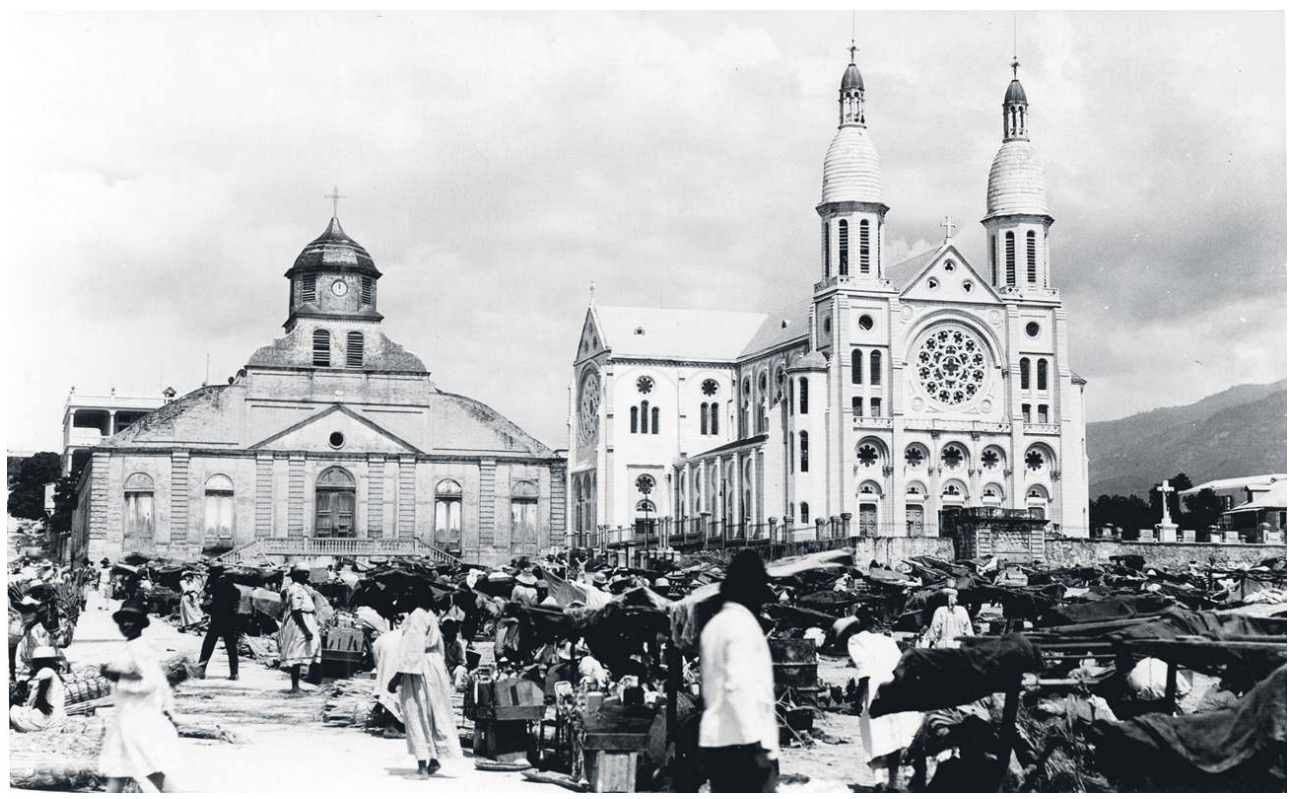

(c) Archives CIDIHCA

Tout est là, en quelque sorte; il suffira d'inverser les propos ou d'écarter certaines considérations en se plaçant dans un univers interprétatif permettant d'admettre la prégnance $\mathrm{du}$ vodou, son statut de religion à part entière, ses fonctions sociales, les valeurs dont il est porteur. C'est ce qu'amorce Dorsainvil en 1912-1913 et que parachèvera Price-Mars. Il n'y parvient pas encore en 1917-1919 dans son analyse socio-historique de la faillite des élites, mais progressivement à travers une série d'études amorcées en 1920. Il découvre un nouvel horizon conceptuel à travers «l'ethnologie du proche » dont les études folkloristes offrent alors le meilleur modèle. L'ouverture à ce nouveau champ lui permet de recueillir et de mettre en perspective les tentatives, les propositions et initiatives antérieures qu'il articule à son projet de réforme sociale énoncé dans $L a$ Vocation de l'élite (1919). Il est désormais à même de renouer avec Trouillot en renversant sa perspective, continuer Dorsainvil en le dépassant. Il est alors armé pour défendre le statut de religion à part entière du vodou et objecter que la possession ne relève pas de la pathologie, ouvrant ainsi «la voie à la théorie moderne de la normalité, culturelle ou sociologique, de la possession $»^{34}$. Price-Mars instaure donc l'ethnologie dans le pays, dont l'implication décisive consiste en une redéfinition de la communauté haïtienne sur de nouvelles bases. La légitimité et les caractéristiques de celle-ci ne tiennent plus uniquement à l'histoire politique mais aussi et surtout, désormais, à sa culture dans toutes ses composantes, y compris et avant tout aux composantes africaines jusque-là refoulées. Certes l'histoire n'est pas répudiée; elle est même essentielle. Yelvington et Magloire y insistent avec raison. Gabriel Debien remarque que Price-Mars « rouvrait par la géographie et l'ethnologie le grand mais difficile cheminement de l'histoire $»^{35}$. Mais, nous l'avons vu, les liens entre histoire et anthropologie sont anciens. Seulement de Madiou à Price-Mars, il y a un changement de perspective. On serait passé du temps de l'histoire (le XIx ${ }^{\mathrm{e}}$ siècle), nourri d'un substrat anthropologique, au temps de l'ethnologie nourrie d'histoire (le $\mathrm{xx}^{\mathrm{e}}$ siècle). Cela ne s'est pas effectué sans tensions, traduites en terme politique. Le président Sténio Vincent (1930-1941) qui négociera la fin de l'occupation états-unienne (1915-1934) choisit « l'histoire » contre l'ethnologie, dans sa 
tentative de réactiver la mémoire officielle nationale. Il entreprend la première grande campagne de restauration des sites et monuments historiques du pays (1931-1934), crée un Musée national (1938), promulgue la première loi de conservation du patrimoine (loi du 23 avril 1940) tout en maintenant (ou en réintroduisant) la prohibition du vodou refusant ainsi la redéfinition du patrimoine commun comme le propose l'ethnologie. Quoi qu'il en soit, celle-ci deviendra la formation discursive dominante du $\mathrm{xx}^{\mathrm{e}}$ siècle haïtien ouvrant un vaste champ de recherche touchant tous les domaines, du droit à la médecine jusqu'à la théologie.

14 Ce tournant ethnologique s'observe un peu partout entre la fin du xixe et le début du xx siècle. En Haïti, il prend un caractère qui le rapproche de ce qui se passe dans d'autres pays de la Caraïbe ou du continent américain ${ }^{36}$. Le situant dans cette mouvance, Bastide dégage la portée de l'œuvre de Price-Mars qui demeure à ses yeux « le pionnier $»^{37}$. Kevin Yelvington apporte une confirmation par un autre biais, qu'il reformule dans ce dossier avec Gérarde Magloire ${ }^{38}$. L'influence de Price-Mars, pas toujours suffisamment reconnue, est pourtant déterminante dans la constitution de l'anthropologie afro-américaniste, par le concours décisif qu'il a apporté à Melville Herskovits. En retour, ce dernier deviendra une référence majeure pour l'école haïtienne d'ethnologie, à l'instar d'autres chercheurs, comme le sera Alfred Métraux.

Métraux publie en 1958 Le Vaudou haitien, aujourd'hui encore un classique sur le sujet. L'ouvrage paraît au bout d'un parcours qui débute en 1941. Christine Laurière, dans son article, retrace le déroulement de ce terrain - l'un des deux plus emblématiques du cheminement de l'ethnologue (pp. 181-208). Initialement motivé par la nécessité d'étudier le vodou en proie à une violente campagne anti-superstitieuse, avant qu'il n'ait complètement disparu, Métraux se retrouvera à l'école de l'anthropologie appliquée, en charge des enquêtes préliminaires à un projet pilote de l'Unesco consacré à l'éducation de base dans la vallée de Marbial. Les enquêtes, bien menées, fructueuses, ont donné lieu à des publications. De jeunes ethnologues haïtiens y ont fait leurs armes. L'un d'entre eux, Rémy Bastien, tirera de ses recherches la matière d'une thèse, soutenue à Mexico, consacrée à la famille; une des rares monographies du genre ${ }^{39}$. Mais le projet de Marbial a échoué, rendant Métraux prudent, sceptique même, sur la portée de l'anthropologie appliquée. 


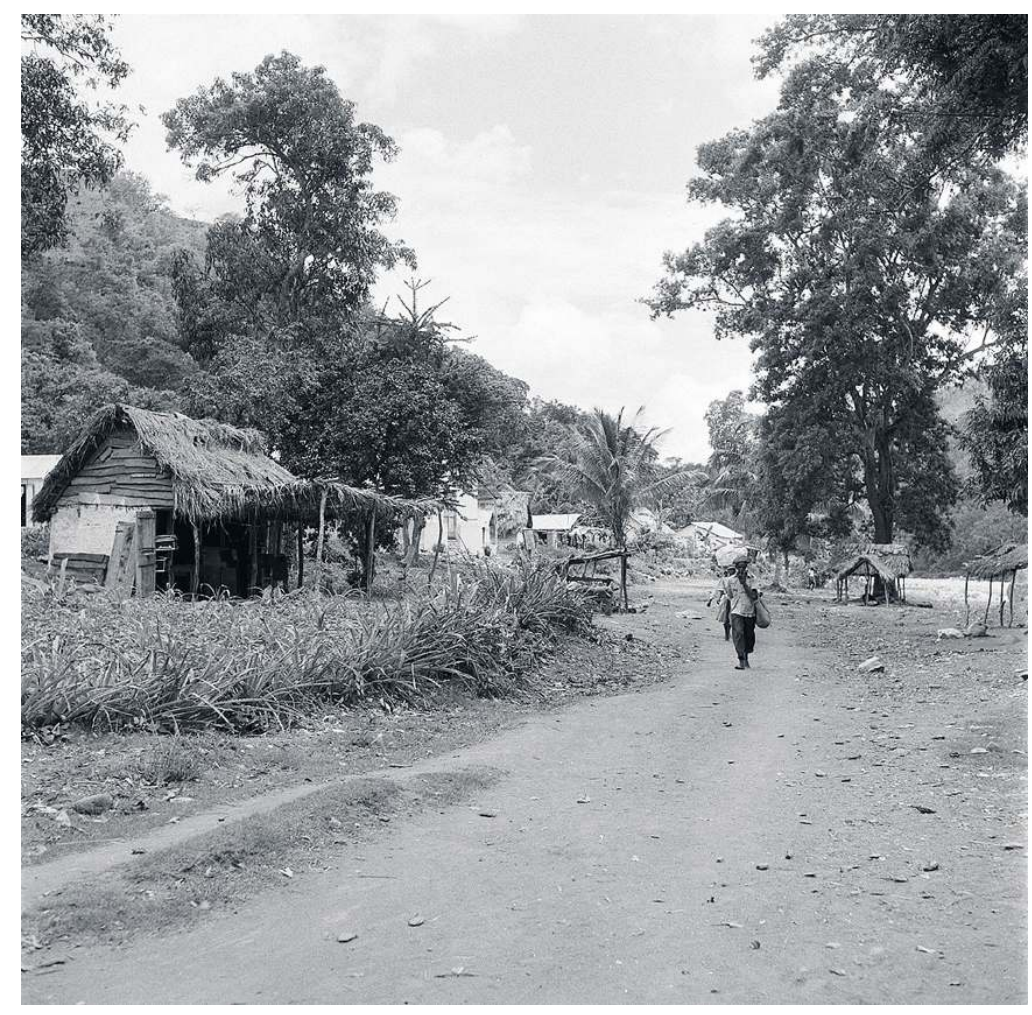

(c) UNESCO

Le rôle joué par Métraux dans la création du Bureau d'ethnologie, ses publications d'où se détache Le Vaudou haitien, son implication dans le projet de Marbial, sa longue présence sur le terrain pendant laquelle il travaille avec de nombreux chercheurs constituent un apport considérable à la consolidation de la recherche ethnographique dans le pays. Apport d'autant plus précieux que sa démarche diffère, par exemple, de celle d'Herskovits en ce qu'elle ne vise pas à identifier et à évaluer des africanismes, mais indique d'autres voies au mouvement ethnologique naissant.

17 Au moment de la première visite de Métraux en Haïti, Jacques Roumain, qui doit certainement sa vocation d'ethnologue à Price-Mars, venait de rentrer d'exil, enrichi de nouvelles vues sur l'anthropologie ${ }^{40}$. Ce qui lui a permis de participer activement au processus d'institutionnalisation de la nouvelle discipline dans le pays en concevant, en 1941, à côté de l'Institut d'ethnologie fondé la même année par Price-Mars, le Bureau d'ethnologie.

L'Institut, devenu une Faculté de l'Université d'État, trouve, en tant que lieu de formation, un complément dans le Bureau, destiné à la recherche. Après le Cercle des Philadelphes emporté par la Révolution, la Société de sciences et de géographie inaugurée en 1886 n'a pas survécu ${ }^{41}$. La Société haïtienne d'histoire et de géographie, fondée en 1923, n'est pas un centre de recherches au même titre que le Bureau. Rachelle Doucet, qui propose une histoire de cet établissement selon une double perspective, intellectuelle et institutionnelle, nous permet d'évaluer ce qui a été conçu et réalisé. De ce panorama, se détache la figure d'Emmanuel C. Paul qui apparaît comme un "maître à penser ", définissant les tâches et missions de l'ethnologie. Il étend sa réflexion au-delà de l'espace national, en plaidant, au Congrès des écrivains et artistes noirs en 1956, pour une 
« anthropologie indigène » dans les sociétés du tiers-monde. En dépit de tout ce qui a pu être produit et apprécié, avant le déclin de l'établissement, on perçoit les difficultés à instaurer les conditions optimales de production des connaissances dans le pays.

Le Bureau, conçu dans le contexte d'un renouveau de l'intérêt pour l'archéologie et d'un désir de sauvetage d'objets cultuels voués à l'autodafé, est dédié à la collecte et à l'étude de la culture matérielle. Il initie de la sorte un courant de recherche qui ne s'est pourtant pas vraiment développé. Ce qui semble confirmer le triomphe, au sein même de l'établissement, de la ligne imprimée par Price-Mars à l'école haïtienne d'ethnologie à partir de sa définition initiale du folklore. La configuration générale de la recherche menée au sein du Bureau, telle que l'a dégagée Raymonde Giordani, semble confirmer cette lecture ${ }^{42}$. Une attestation supplémen- taire est apportée par R. Doucet, qui montre que l'établissement a véritablement pris sa forme sous l'administration de Lorimer Denis et d'Emmanuel C. Paul, disciples de Price-Mars. Ce peu d'intérêt pour l'étude de la culture matérielle semble expliquer, au moins en partie, que l'école haïtienne d'ethnologie n'a pas fait de la création plastique une matière de recherches (cf. Célius, pp. 71-94). On comprend que la plastique vodou elle-même demeure un domaine encore peu exploré et qu'une collection comme celle de Marianne Lehmann, présentée ici par Rachel BeauvoirDominique (pp. 57-70), suscite interrogations et même controverses, tout en ouvrant de nouvelles perspectives appelées à bouleverser la représentation, qui s'est construite jusqu'à maintenant, du monde de la création plastique d'Haïti.

\section{Mardi-Gras à Port-au-Prince}

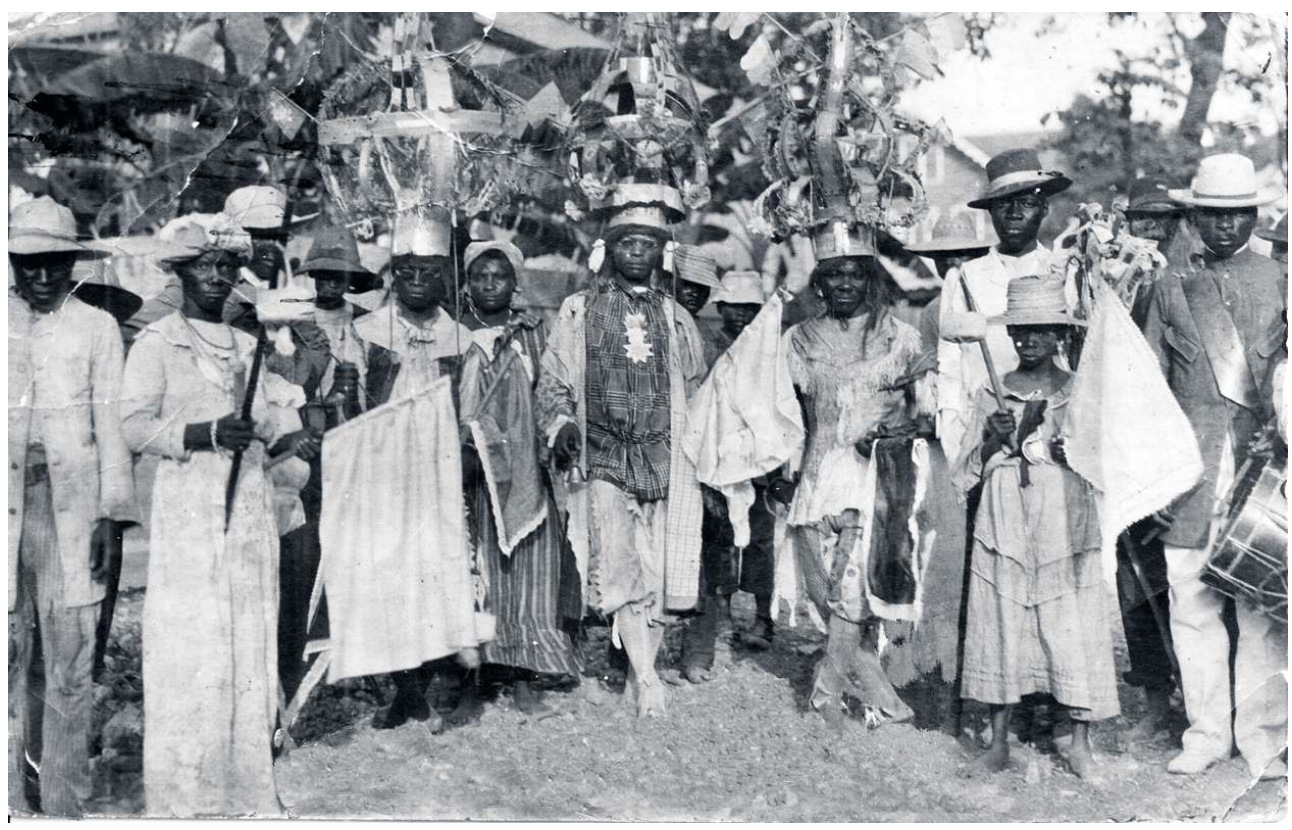

(C) Archives CIDIHCA

Ce point et sans aucun doute bien d'autres expliquent que le vodou apparait encore, tant aux yeux de Rachel Beauvoir-Dominique que de Laënnec Hurbon, peu connu, en dépit de sa centralité dans le tournant ethnologique. Il en est de même du statut du vodou dans la société. L. Hurbon en retrace à grands traits l'histoire depuis la période coloniale (pp. 153-164), et remarque qu'aujourd'hui encore rien n'est réglé, malgré des signes évidents de changement dans les représentations. Si le vodou a finalement acquis le statut de religion dans les années 1930, il est longtemps resté juridiquement prohibé, jusqu'à la 
suppression de toute pénalisation par la Constitution de 1987, après la vague de destruction de 1986. La diabolisation du vodou est constamment ravivée, notamment par les cultes protestants; de même est encore vivace la velléité de manipulation politique, comme le prouve, selon l'analyse de L. Hurbon, l'arrêté pris en avril 2003 en faveur du vodou par le gouvernement de Jean-Bertrand Aristide. Kate Ramsey s'intéresse également à cette question de la pénalisation du vodou, en se focalisant sur les années 1930 et 1940 (pp.165-180). Elle montre comment pénalisation et processus de folklorisation du vodou sont associés au moment de l'avènement de l'école haïtienne d'ethnologie. Ce qui lui permet de soutenir qu'il n'y a nulle incompatibilité ou ambiguïté à ce que le gouvernement d'Élie Lescot puisse à la fois soutenir la campagne antisuperstitieuse et accepter que soit créé par décret le Bureau d'ethnologie. Elle montre surtout ici ce que les chercheurs ont souvent occulté: que les premières enquêtes du mouvement ethnographique se sont déroulées dans un contexte de prohibition du vodou, ce qui a incontestablement influé sur la forme et le contenu des résultats, bien que, par ailleurs, une telle situation ait stimulé le développement des études.

21 En dépit de la position centrale, dominante même, du vodou, il convient de signaler la diversité thématique, mais aussi méthodologique et théorique, marquant le développement des études ethnographiques. Parce qu'il s'attache plus particulièrement à reconsidérer le tournant ethnologiquedans sa globalité, le dossier ne rend pas assez justice à cette diversité. Nombre de figures marquantes auraient mérité qu'on discute leur œuvre. Louis Mars par exemple. Suzanne Comhaire-Sylvain aussi, dont Kathleen Gyssels offre ici un rapide aperçu en analysant un des contes que la première a recueillis (pp. 243-248).

Des études traitant de l'anthropologie avant Firmin, de la période nationale à l'époque coloniale, auraient apporté des éclairages significatifs. Un bilan des études menées ces vingt dernières années aurait également permis d'apprécier la dynamique à l'œuvre audelà du temps d'institutionnalisation de l'ethnologie en Haïti. La contribution de Chantal Collard, au travers de l'analyse du fonctionnement de l'adoption internationale telle qu'elle se déroule en Haïti, ouvre des perspectives à l'ethnologie d'aujourd'hui (pp. 209-224) et constitue de ce fait un bon exemple des nouveaux terrains à explorer dans ce pays.

\section{NOTES}

1. André Marcel d'Ans, "Préface", in Rémy Bastien, Le Paysan haïtien et sa famille. Vallée de Marbial, Paris, Karthala, 1985 [1951], p. 9.

2. Louis Sala-Molins, Le Code noir, ou le calvaire de Canaan, Paris, Presses universitaires de France (« Quadrige »), 2002 (1re éd. 1987).

3. Jean-Luc Bonniol, «La couleur des hommes, principe d'organisation sociale. Le cas antillais », Ethnologie française, 1990, 20 (4), pp. 410-418; id., La Couleur comme maléfice. Une illustration créole de la généalogie des Blancs et des Noirs, Paris, Albin Michel (« Bibliothèque de synthèse »), 1992.

4. Margaret Hodgen, Early Anthropology in the Sixteenth and Seventeenth Centuries, Philadelphia, University of Pennsylvania Press 1964 ; Anthony Pagden, The Fall of Natural Man: the American Indian and the Origins of Comparative Ethnology, Cambridge, Cambridge University Press, 1982 ; Michel-Rolph Trouillot, "The Caribbean Region: An Open Frontier in Anthropology Theory ", Annual Review of Anthropology, 1992, 21, pp. 19-42.

5. Trouillot, 1992, op. cit. 
6. Catts Pressoir, Ernst Trouillot \& Hénock Trouillot, Historiographie d'Haïti, Mexico, Instituto panamericano de geografia e historia, Comision de historia 66 (« Historiografias » I-168), 1953.

7. James E. McClellan III, Colonialism and Science. Saint Domingue in the Old Regime, BaltimoreLondon, The Johns Hopkins University Press, 1992.

8. Élie Moreau de Saint-Méry, Description topographique, physique, civile, politique et historique de la partie française de l'isle de Saint-Domingue, éd. par Blanche Maurel \& Étienne Taillemite. Paris, Société française d'histoire d'outre-mer («Bibliothèque d'histoire d'outre-mer »), 1984 [1797], 3 vol.

9. Jean Price-Mars, Le Bilan des études ethnologiques en Haïti et Le cycle du Nègre, Port-au-Prince, Imprimerie de l'État, 1954, p. 16.

10. Thomas Madiou, Histoire d'Haïti, I. 1492-1799, Port-au-Prince, H. Deschaps, 1989 (1 $1^{\text {re }}$ éd. 1847), pp. VIII-IX.

11. Ghislaine Géloin, "Introduction", in Anténor Firmin, De l'égalité des races humaines (Anthropologie positive), Paris, L'Harmattan, 2004 [1885], pp. XI-XXVII.

12. Cette chercheuse occupe une place importante dans la redécouverte de Firmin et aussi dans sa découverte par le monde anglo-saxon. Associée à la traduction anglaise de De l'égalité des races humaines (The Equality of the Human Races, New York, Garland Press 2000), elle en a aussi rédigé l'introduction. À l'occasion de la parution de cet ouvrage, elle a en outre co-organisé le colloque, «Rediscovering Antenor Firmin, Pioneer of Anthropology, Pan-africanism and Post-colonial Studies ", à Rhode Island College, Providence, 1-2 juin 2001.

13. Robert Bernasconi, de l'University of Memphis, place Firmin aux côtés des principales figures de l'anthropologie raciale du XIX ${ }^{\mathrm{e}}$ siècle, tels Georges Pouchet, Paul Broca, Armand de Quatrefages, Carl Vogt, Paul Tropinard, par exemple, dont il ré-édite d'ailleurs les œuvres. De l'égalitré des races humaines est le huitième volume. Cf. Robert Bernasconi, ed., Race and Anthropology, Bristol-London, Thoemmes Continuum, 2003, 9 vol.

14. Roger Gaillard, "Introduction", in Jean Price-Mars, Ainsi parla l'oncle, Port-au-Prince, Imprimeur II, 1998 [1928], pp. XIV - XVI.

15. Ghislain Gouraige, "Le Dr Price-Mars chef d'école et critique littéraire ", in Emmanuel C. Paul \& Jean Fouchard, s. dir., Témoignages sur la vie et l'œuvre du Dr Jean Price-Mars 1876-1956, Portau-Prince, Imprimerie de l'État, 1956, pp. 53-65.

16. Antoine Innocent, Mimola ou l'histoire d'une cassette: petit tableau de mours locales, Port-auPrince, V. Valcin Imp., 1935 (1re éd. 1906).

17. J. C. Dorsainvil, Vodou et névrose, Port-au-Prince, Éd. Fardin, 1975 (1 ${ }^{\text {re }}$ éd. 1931).

18. Dorsainvil, 1975, op. cit., p. 9.

19. Dorsainvil, op. cit., p. 17.

20. Jean Chrisostome Dorsainvil, médecin, a été chef de division du Département de l'Agriculture, puis celui de l'Instruction publique et enfin Inspecteur de l'enseignement rural. Ces différentes fonctions lui ont offert la possibilité de sillonner le pays.

21. Dorsainvil, 1975, op. cit., p. 21.

22. Ibid., p. 143.

23. Ibid., p. 147.

24. Ibid., p. 150.

25. Ibid., p. 155.

26. Ibid., p. 163.

27. Cf. la bibliographie des œuvres de Comhaire-Sylvain préparée par P. Salmon, «ComhaireSylvain (Suzanne Marie Joséphine Anne Marguerite Antoinette Rose) » in Biographie belge d'outremer, Bruxelles, ARSOM, Commission de la Biographie belge d'outre-mer, 1998, vol. VIII, pp. 51-56.

28. Suzanne Comhaire-Sylvain, Le Créole haïtien: morphologie et syntaxe, Weteren (Belgique) Imprimerie De Meester / Port-au-Prince (Haïti), chez l'Auteur, 1936, p. 178. 
29. Félix Prudent, «L'africanité dans la genèse créole. Science et idéologie d'un lignage ", Études Créoles, 1986, 9 (1), pp. 151-168; Robert Chaudenson, Des îles, des hommes, des langues : essai sur la créolisation linguistique et culturelle, Paris, L'Harmattan, 1992 ; id., Les créoles, Paris, PUF (« Que saisje?»), 1995.

30. Sur le parcours de Louis Mars et pour une bibliographie de ses œuvres, cf. Bulletin du Bureau national d'ethnologie, 1986, nos 1 et 2 ; Emerson Douyon, La Crise de possession dans le vaudou haïtien, Montréal, Université de Montréal, thèse de doctorat, 1964 ; Jeanne Philippe, Classes sociales et maladies mentales en Haïti, Port-au-Prince, Presses nationales, 1975 ; Willy Apollon, Le Vaudou : un espace pour les « voix », Paris, Éditions Galilée, 1976. 2.

31. Georges Devereux, préface (pp. XI-XVI) à Louis Mars, La Crise de possession. Essais de psychiatrie comparée, Port-au-Prince, Imprimerie de l'État («Bibliothèque de l'Institut d'Ethnologie »), 1955 [1 $1^{\text {re }}$ éd. 1946].

32. Price-Mars, 1954, op. cit., p. 17.

33. D. Trouillot, Esquisse ethnographique. Le Vaudoun : aperçu historique et évolutions, Port-auPrince, Imprimerie R. Ethéard, 1885.

34. Roger Bastide, «Le Dr Jean Price-Mars et le vodou », in Emmanuel C. Paul \& Jean Fouchard, s. dir., Témoignages sur la vie et l'œuvre du Dr Jean Price-Mars 1876-1956, op. cit., p. 200.

35. Gabriel Debien, «Trente ans d'une œuvre historique », in ibid., p. 128.

36. Cf. Erwan Dianteill, « Una filiación problemática : Dos generaciones de antropólogos en Cuba y Haití », Del Caribe, 2002, 37 : 28-37 ; Marion Aubrée \& Erwan Dianteill, « Misère et splendeur de l'afro-américanisme. Une introduction ", Archives de Sciences sociales des Religions, 2002, 117 : 5 -15. 37. Roger Bastide, op. cit.

38. Kevin Yelvington, "The Invention of Africa in the Caribbean: Political Discourse and Anthropological Praxis, 1920-1940», contribution au School of American Research Advanced Seminar, Santa Fe, NM, 10-15 avril 1999.

39. Rémy Bastien, op. cit.

40. André Marcel d'Ans, "Jacques Roumain et la fascination de l'ethnologie ", in Jacques Roumain, Euvres complètes. Éd. coordonnée par Léon-François Hoffmann. Madrid, Allca XXUnesco (« Archivos » 58), 2003, pp. 1378-1428.

41. Jean Liautaud (texte recueilli par), Acte de Constitution et Statuts de la Société de sciences et de géographie d'Haïti (2 juin 1886), Revue de la Société haïtienne d'Histoire et de Géographie, 2001, 208, pp. 51-55.

42. Raymonde Giordani, L'Anthropologie indigène: état de la question, essai d'application, Québec, Université Laval, mémoire de maîtrise, 1987.

\section{INDEX}

Mots-clés : ethnologie et politique, ethnologie haïtienne, histoire de l'anthropologie Keywords : ethnology and politics, haitian ethnology, history of anthropology 


\section{AUTEUR}

\section{CARLO AVIERL CÉLIUS}

Université Laval, Chaire d'histoire comparée de la mémoire-CÉLAT, Québec, Canada, carloa.celius.1@ulaval.ca 